\title{
Risk Factors and Prognostic Impact of the Completion of Adjuvant Chemotherapy with Oxaliplatin Plus Oral Fluorouracil for AGC After D2 Gastrectomy
}

\section{Meng-Ting Pan}

Taizhou Hospital of Zhejiang Province

\section{Yu-Qiang Huang}

Fujian Medical University

\section{Yan-Yun Wu}

The First Affiliated Hospital of Xiamen University

Ming-Xu Luo

Xiamen Humanity Hospital

Yu-Fei Ji

The First Affiliated Hospital of Xiamen University

\section{Zha-Xi Baima}

Xiamen University

\section{Hui-Yao Lin}

Fujian University of Traditional Chinese Medicine

Bo Chen ( $\nabla$ chenbo7892@xmu.edu.cn )

First Affiliated Hospital of Xiamen Universtiy https://orcid.org/0000-0002-2796-8201

\section{Research Article}

Keywords: Advanced gastric cancer, Adjuvant chemotherapy, Completion rate of chemotherapy, Prognosis

Posted Date: October 25th, 2021

DOl: https://doi.org/10.21203/rs.3.rs-966715/v1

License: (1) This work is licensed under a Creative Commons Attribution 4.0 International License. Read Full License 
1 Risk factors and prognostic impact of the completion of 2 adjuvant chemotherapy with oxaliplatin plus oral

3 fluorouracil for AGC after D2 gastrectomy

4

5 Meng-Ting Pan ${ }^{1}$, Yu-Qiang Huang ${ }^{2}$, Yan-Yun $\mathrm{Wu}^{3}$, Ming-Xu Luo ${ }^{4}$, Yu-Fei Ji ${ }^{3}$, Zha-Xi

6 Baima $^{5}$, Hui-Yao Lin ${ }^{6}$, Bo Chen ${ }^{3}$

7

$8 \quad{ }^{1}$ Department of Hematology and Oncology, Taizhou Hospital of Zhejiang Province

9 affiliated to Wenzhou Medical University, Taizhou, China

${ }^{2}$ Department of Clinical Medicine, Fujian Medical University, Fuzhou, China

${ }^{3}$ Department of Gastrointestinal Surgery, Xiamen Cancer Center, The First Affiliated Hospital of Xiamen University, Xiamen, China

${ }^{4}$ Department of Gastrointestinal Surgery, Xiamen Humanity Hospital, Xiamen, China

${ }^{5}$ Department of Medical College Xiamen University, Xiamen University, Xiamen, China

${ }^{6}$ Department of College of Integrated Traditional Chinese and Western Medicine, Fujian University of Traditional Chinese Medicine, Fuzhou, China

\section{Equal contributors:}

Meng-Ting Pan, Yu-Qiang Huang contributed equally to this work.

\section{Correspondence author:}


Bo Chen, Department of Gastrointestinal Surgery, Xiamen Cancer Center, The First Affiliated Hospital of Xiamen University, No.55 Zhenhai Road, Xiamen 361003, China. E-mail address: chenbo7892@xmu.edu.cn. Tel No: +86 19959280781.

\section{Abstract}

Background: To analyze the risk factors and prognostic impact of the completion of adjuvant chemotherapy with oxaliplatin plus oral fluorouracil for advanced gastric cancer (AGC) after D2 gastrectomy.

Methods: A total of 234 patients with AGC who underwent D2 gastrectomy and received oxaliplatin plus oral fluorouracil adjuvant chemotherapy between January 1, 2012 to January 1, 2021 in the First Affiliated Hospital of Xiamen University were identified (203 cases with SOX regime;31 cases with XELOX regime) and their clinicopathological information was retrospectively analyzed. The tolerability and safety of oxaliplatin plus oral fluorouracil therapy were analyzed. Patients were divided into high completion group $(\mathrm{n}=135)$ and low completion group $(\mathrm{n}=99)$ according to whether they had completed 6 cycles of adjuvant combined chemotherapy. Relevant risk factors and the prognostic impact of the completion rates of chemotherapy were analyzed.

Results: 99 patients have completed more than 6 cycles of adjuvant chemotherapy with oxaliplatin plus oral fluorouracil and the chemotherapy completion rate was $42.31 \%$. Chemotherapy completion was correlated with age $(P=0.000)$ and the extent of gastric resection $(P=0.039)$. Age was independent risk factor of chemotherapy completion 
$(O R, 2.487 ; 95 \% C I, 1.449-4.268 ; P=0.001)$. As of January 1,2021, 124 patients were

followed up for more than three years, the three-year survival rate $(86.9 \%$ vs $68.8 \% ; P$ $=0.02)$ were significantly different between two groups, and the differences of $\mathrm{pN}$ stage, pTNM stage and nerve invasion were also statistically significant $(P<0.05$, respectively). Cox regression analysis showed that $\mathrm{pN} 3$ stage $(H R, 2.645 ; 95 \% C I, 1.138$ 6.146; $P=0.024)$ was independent prognostic factor about poor survival.

Conclusions: Oxaliplatin plus fluorouracil oral adjuvant chemotherapy was safe and tolerable. Advanced age and total gastrectomy were risk factors for chemotherapy completion. The key to improve the prognosis of patients with AGC after D2 gastrectomy was to improve the completion rate of adjuvant chemotherapy.

Keywords: Advanced gastric cancer; Adjuvant chemotherapy; Completion rate of chemotherapy; Prognosis

\section{Background}

Gastric cancer was one of the most common malignant tumors in the world. In 2018, there were 1 million new cases of gastric cancer, and nearly 800 thousand people died of gastric cancer [1]. The incidence rate of gastric cancer has increased significantly in East Asia countries such as Japan, Korea and China [1, 2]. The detection rate of early asymptomatic gastric cancer was high in Japan and Korea [3-5]. However, more than $80 \%$ of Chinese patients with gastric cancer were diagnosed as advanced gastric cancer ( $A G C$ ) for the first time $[6,7]$. 
Radical resection was the only possible way to cure AGC [8], including standard gastrectomy and D2 lymphadenectomy [7-9]. But the recurrence rate of patients with AGC after radical resection was more than $60 \%$, and more than $40 \%$ of patients relapsed within 2 years [10]. Postoperative adjuvant chemotherapy was widely used in Asia. The adjuvant chemotherapy based on fluorouracil can significantly improve the curative effect and reduce recurrence, and the 5-year survival rate can be increased by nearly 50\% [11]. SOX regimen and XELOX regimen were the most commonly used adjuvant chemotherapy for AGC after D2 radical gastrectomy in China $[7,12-25]$.

The prognosis of patients with AGC was still not optimistic, even if they receive comprehensive treatment based on surgery [3]. The optimization of adjuvant chemotherapy was the key to delay tumor progression or recurrence and help patients maintain good quality of life and prolong survival time. IDEA study emphasizes the importance of sufficient duration of adjuvant chemotherapy for colon cancer [26]. JCOG1104 study showed that the 3-year RFS of patients with stage III AGC who received one-year S-1 single agent chemotherapy after radical resection was higher than that of patients receiving half a year of chemotherapy [27]. The second post hoc analysis of the classic study emphasized that patients with stage II - III advanced gastric cancer who received more than 6 cycles of XELOX postoperative adjuvant chemotherapy had a significantly better prognosis [23]. The ACTS-GC study showed that only $65.8 \%$ of patients completed one-year S-1 single agent adjuvant chemotherapy [12], and only 67\% of patients in the CLASSIC study completed 8 cycles of XELOX adjuvant 
chemotherapy[22]. It can be seen that the completion rate of adjuvant chemotherapy was relatively low in previous phase II or III clinical studies. Prolonging the time of adjuvant chemotherapy and improving the completion rate of postoperative chemotherapy were the key to improve the prognosis of patients with AGC after radical operation.

\section{Methods}

This study has been approved by ethics committee of The First Affiliated Hospital of Xiamen University. The clinicopathological information of patients with AGC who underwent D2 gastrectomy and received oxaliplatin plus oral fluorouracil adjuvant chemotherapy between January 1, 2012 to January 1, 2021 in the First Affiliated Hospital of Xiamen University were collected retrospectively. According to the inclusion criteria and exclusion criteria, 234 eligible cases were successfully included. Inclusion criteria: (1) No metastasis was found by preoperative examination and intraoperative abdominal exploration; (2) D2 radical resection was performed by the surgeon of our hospital under the assistance of laparotomy or laparoscopy. The postoperative pathological report confirmed that it was advanced gastric adenocarcinoma with negative upper and lower margins; (3) The patient received postoperative adjuvant chemotherapy in our hospital. Before the first chemotherapy, the patient's self-care skill, bone marrow and important organ function were basically normal; (4) The postoperative adjuvant chemotherapy regime was L-OHP plus oral 

preoperative chemotherapy or preoperative radiotherapy; (3) D1 / D1 + lymph node dissection; (4) Chemotherapy particles were placed in the abdominal cavity during operation; (5) The postoperative pathological report could not confirm that the tumor was adenocarcinoma or combined with squamous cell carcinoma, or the upper and lower margins were positive; (6) The patient received postoperative radiotherapy; (7) The patients died within 30 days after operation. obtain relevant information. The life span started from the date of the first day after operation, ended in the dead day or the last follow-up date, and the deadline date of follow-up was January 1, 2021. Statistical analysis was performed using Stata13.0 software. Single factor analysis of counting data was used $\chi^{2}$ test. Multivariate analysis used binary logistic regression model. The case (\%) was used to perform binary classification data. The time-point survival rate and overall survival rate were calculated. The cumulative mortality was estimated using the Kaplan-Meier method and the survival curve was obtained. Univariate analyzed was performed by Log-rank test, statistically significant factors were selected and analyzed using a multivariate COX proportional hazards model to explore factors affecting the prognosis of patients with AGC. The hazard ratio (HR) was estimated using a 95\% confidence interval (CI), $\mathrm{p}<0.05$ was considered statistically significant.

\section{Results}




\section{Case data introduction}

According to the inclusion and exclusion criteria, a total of 234 patients with

AGC who underwent D2 gastrectomy and received oxaliplatin plus oral fluorouracil adjuvant chemotherapy were identified (203 cases with SOX regime; 31 cases with XELOX regime). Their clinicopathological information were shown in Table 1.

\section{Tolerability and safety of adjuvant chemotherapy}

\section{Tolerability}

99 patients have completed more than 6 cycles of adjuvant chemotherapy with LOHP plus oral fluorouracil and the chemotherapy completion rate was $42.31 \%$. 41 patients $(30.37 \%)$ continued oral fluorouracil treatment after interrupting L-OHP treatment. The average interval between the first chemotherapy and operation was 33.98 days. 203 patients received SOX chemotherapy, the average dose intensity of L$\mathrm{OHP}$ and S-1 were $117.13 \mathrm{mg} / \mathrm{m}^{2}$ and $35.31 \mathrm{mg} / \mathrm{m}^{2}$ respectively. 172 patients experienced delay, the average time interval two cycle of chemotherapy were 27.97 days. 31 patients received XELOX chemotherapy, the average dose intensity of L-OHP and CAPE were $119.37 \mathrm{mg} / \mathrm{m}^{2}$ and $923.88 \mathrm{mg} / \mathrm{m}^{2}$ respectively. 27 patients experienced delay, the average time interval two cycle of chemotherapy were 27.95 days.

\section{Safety}

192 patients $(82.05 \%)$ suffered from adverse events. Grade 2 or higher adverse events occurred in 108 patients (46.15\%) 17 patients developed obvious gastrointestinal adverse reactions, mainly nausea and vomiting. Serious peripheral neuritis and serious 
oral ulcer was observed in one patient respectively. Hematological toxicities of all grades occurring in at least 10 patients were listed in Table 2. Anemia occurred in 160 patients (68.38\%), it was the most common hematological toxicities of all grades, but grade 2 or higher events were observed in only 20 patients $(8.55 \%)$. The most common hematological adverse reaction of grade 2 or higher was neutropenia, which occurred in 54 patients (23.08\%), and grade 3 neutropenia occurred in 19 patients $(8.12 \%)$. Grade 4 thrombocytopenia occurred in one patient.

\section{Correlation analysis for chemotherapy completion}

\section{Univariate analysis of chemotherapy completion}

The chemotherapy completion rate of L-OHP plus oral fluorouracil for more than 6 cycles was significantly correlated with the age $(P=0.000)$ and the extent of gastric resection $(P=0.039)$ (Table 3$)$.

\section{Multivariate analysis of chemotherapy completion}

The significant items of univariate analysis were introduced into logistic regression model. In multivariable regression models, age was independent risk factor of chemotherapy completion $(O R, 2.487 ; 95 \% C I, 1.449-4.268 ; P=0.001)$. Patients with distal gastrectomy were 1.623 times more likely to complete more than 6 cycles of adjuvant chemotherapy than patients with total gastrectomy $(\mathrm{P}=0.078,95 \% \mathrm{CI}, 0.947$ -
2.781). (Table 4) 


\section{Survival analysis}

173

174

\section{Comparison of survival prognoses between high completed group and low}

\section{completed group}

124 patients with AGC who underwent D2 gastrectomy and received oxaliplatin plus oral fluorouracil adjuvant chemotherapy before January 1, 2018. Median followup time was 43.9 months. The survival rate was calculated by Kaplan-Meier method.

The 1-year, 2-year and 3-year OS rate of patients were 96.6\% (S $\bar{X}=0.017), 83.9 \%$ $(S \bar{X}=0.035)$ and $76.4 \%(S \bar{X}=0.041)$ respectively (Fig. $)$. As shown in Table 5, there was significant difference in OS rate between high completion group and low completion group (1-year OS rate, $100 \%$ vs $94.2 \%, P=0.04$; 2-year OS rate, $93.6 \%$ vs $76.9 \%, P=$ 0.01 ; 3-year OS rate, $86.9 \%$ vs $68.8 \%, P=0.02$ ). The Kaplan-Meier curves for the two groups were shown in Fig.2. A significant separation between the two groups was observed.

\section{Univariate analysis of clinicopathological factors affecting three-year survival}

rate

The 3-year OS rate was calculated by Kaplan-Meier method, the survival curve was drawn, and the Log-rank test was performed. The differences of $\mathrm{pN}$ stage, $\mathrm{pTNM}$ stage and nerve invasion were also statistically significant $(P<0.05$, respectively) (Fig.3-5). The above factors affected the prognosis of patients with AGC who underwent D2 gastrectomy and received L-OHP plus oral fluorouracil adjuvant chemotherapy (Table 6). 


\section{Multivariate analysis of prognostic factors}

As shown in Table 7, Cox regression analysis showed that pN3 stage $(H R, 2.645$; 95\%CI,1.138-6.146; $P=0.024)$ was independent prognostic factor about poor survival. Nerve invasion was a risk factor affecting the prognosis of patients $(H R, 2.260 ; 95 \% C I$, 0.266-1.092; $P=0.057)$. Completion of more than 6 cycles of adjuvant chemotherapy was a favorable factor affecting the prognosis of patients $(H R, 0.539 ; 95 \% C I, 0.266-$ $1.092 ; P=0.086)$

\section{Discussion}

This study suggests that the completion of L-OHP plus oral fluorouracil adjuvant chemotherapy after D2 radical gastrectomy was closely related to the prognosis of patients. Improving the completion rate of adjuvant chemotherapy is the key to improve the prognosis of patients. In this study, the completion rate of combined chemotherapy for patients with more than 6 cycles was only $42.31 \%$. The completion rate of SOX regimen and XELOX regimen was 42.36\% (86/203) and 41.94\% (13/31) respectively, which were lower than that of previous studies $[16,22]$. However, the safety of chemotherapy was similar to previous studies and the adverse reactions of chemotherapy were also controllable. L-OHP plus oral fluorouracil adjuvant chemotherapy was safe and tolerable.

The results showed that the completion rate of chemotherapy in elderly patients was significantly reduced. Most patients with AGC in China were aged from 60 to 70[28]. The incidence of grade 3 or above adverse reactions in elderly patients 
with AGC was significantly higher than that in young patients [29]. The key of treatment for elderly patients with AGC was to ensure that the expected benefit was higher than the expected risk. There were many basic diseases in elderly patients, so the individual drug treatment history of the elderly was relatively complex. Moreover, the elderly group has poor state of ability, and the immunity was reduced, and it was unable to withstand high-dose combined chemotherapy with multiple drugs. It can be considered to adjust the mode of chemotherapy administration $[16,30,31]$, reduce the toxicity of chemotherapy $[17-19,32]$ and reduce the types of chemotherapy drugs [33, 34] for improving the completion rate of adjuvant chemotherapy in elderly patients with AGC after radical resection. Elderly patients need to formulate individualized treatment. In addition, in order to find more asymptomatic patients with early gastric cancer and improve their life quality, gastroscopy should be actively promoted in our country [3537].

The results showed that the completion rate of chemotherapy in patients with AGC underwent total gastrectomy was significantly reduced. The standard surgical treatment of AGC was distal gastrectomy or total gastrectomy [7]. In this study, the tumors were located in the lower third of the stomach in 121 patients, of which $12.40 \%$ of patients underwent total gastrectomy. Total gastrectomy has many adverse factors, such as prolonging the postoperative hospital stay, prolonging the time of postoperative low calorie intake, increasing the long-term nutritional sequelae and reducing the quality of life of patients [38-42]. The results also showed that the nutritional status of patients who underwent total gastrectomy was poor. $58.41 \%$ of the patients lost more than $7 \%$ 
of their weight after total gastrectomy, and $28.32 \%$ of patients even more than $10 \%$.

The time for patients with total gastrectomy to receive the first adjuvant chemotherapy was significantly delayed, and $15.04 \%$ of patients had an interval of more than 6 weeks.

Distal gastrectomy was recommended for patients with lower third AGC when the proximal margin distance can be ensured. The choice of total gastrectomy or distal gastrectomy for the middle third of AGC has not yet been decided [43, 44]. For patients with AGC who cannot ensure the proximal margin distance and need total gastrectomy, perioperative chemotherapy can be considered to reduce the tumor stage, strive for partial gastrectomy, improve the completion rate of chemotherapy, improve the overall treatment effect and improve the life quality of patients [9, 25, 45-47]. further improve the prognosis of patients with high lymph node metastasis.

The results showed that $\mathrm{pN} 3$ stage was independent prognostic factor about poor survival of patients with AGC who underwent D2 gastrectomy and received L-OHP plus oral fluorouracil adjuvant chemotherapy. High lymph node metastasis was an important predictor of poor prognosis in patients with AGC [48-51]. SP regimen preoperative chemotherapy was safe and effective for patients with high lymph node metastasis [52-55]. The results of FLOT4 study were better than the previous standard treatment [56-58]. The Chinese study confirmed that it was also safe and feasible for Chinese population to receive perioperative FLOT chemotherapy[59]. Patients with high lymph node metastasis can choose preoperative SP chemotherapy or perioperative FLOT chemotherapy, and randomized controlled clinical trials can be carried out to 


\section{Conclusions}

L-OHP plus oral fluorouracil adjuvant chemotherapy was safe and tolerable.

261 Advanced age and total gastrectomy were risk factors for chemotherapy completion.

262 The key to improve the prognosis of patients with AGC after D2 gastrectomy was to

263 improve the completion rate of adjuvant chemotherapy.

264

265 List of abbreviations

266

\begin{tabular}{ll}
\hline Abbreviation & \\
\hline AGC & Full name \\
AJCC/UICC & American Joint Committee on Cancer/ International Union Against \\
& Cancer \\
SOX & S-1 plus Oxaliplatin \\
XELOX & Capecitabine plus Oxaliplatin \\
DS & Docetaxel plus S-1 \\
SP & S-1 plus Cisplatin \\
OS & Overall Survival \\
DFS & Disease Free Survival \\
RFS & Relapse Free Survival \\
CA724 & Carbohydrate Antigen 724 \\
&
\end{tabular}


Healthcare Information and Management Systems Society Electronic

HIMSS EMRAM Medical Record Adoption Model

AST aspartate aminotransferase

ALT alanine aminotransferase

TBIL total bilirubin,

ALP alkaline phosphatase

GGT $\quad \gamma$-glutamyl transpeptidase

268 Declarations

269 Ethics approval and consent to participate: This study has been approved by

270 ethics committee of The First Affiliated Hospital of Xiamen University.

271 Consent for publication: All presentations of case reports must have consent for

272 publication.

273 Availability of data and materials: The datasets used and/or analysed

274 during the current study are available from the corresponding author on

275 reasonable request.

276 Competing interests: The authors have declared no conflicts of interest.

277 Funding: The Natural Science Foundation of Fujian Province (Grant

278 number:2018J01377); and the Xiamen Huimin Project of Science and Technology

279 (Grant number: 3502Z20174072). The funders had no role in the study design, 
data collection and analysis, decision to publish, or preparation of the manuscript.

Authors' contributions: Meng-Ting Pan and Yu-Qiang Huang collected and analyzed/interpreted data and drafted the manuscript. Zha-Xi Baima, Hui-Yao Lin and Yu-Fei Ji collected data and revised the manuscript. Ming-Xu Luo analyzed/interpreted data, performed statistical analyses, and revised the manuscript. Bo Chen designed the study, analyzed/interpreted data, and revised the manuscript. All authors read and approved the final manuscript. staff who participated in this study.

\section{References}

1. Bray F, Ferlay J, Soerjomataram I, Siegel RL, Torre LA, Jemal A: Global cancer statistics 2018: GLOBOCAN estimates of incidence and mortality worldwide for 36 cancers in 185

2. Chen W, Zheng R, Baade PD, Zhang S, Zeng H, Bray F et al: Cancer statistics in China, 2015. CA-a cancer journal for clinicians 2016; 66:115-32. for gastric cancer in Japan. World J Gastroenterol 2006; 12:4873-4. 
303 5. Kula ZK, Zegarski W, Jozwicki W: Diagnosis and treatment of early gastric cancer: experience of one center. Przeglad gastroenterologiczny 2018; 13:200-5.

305 6. Yang K, Hu JK: Gastric cancer treatment: similarity and difference between China and Korea. Translational Gastroenterology and Hepatology 2017; 2.

7. Wang FH, Shen L, Li J, Zhou ZW, Liang H, Zhang XT et al: The Chinese Society of Clinical Oncology (CSCO): clinical guidelines for the diagnosis and treatment of gastric cancer. Cancer Communications 2019; 39:10.

8. Japanese Gastric Cancer Association: Japanese gastric cancer treatment guidelines 2018 (5th edition). Gastric Cancer 2020:21.

9. Waddell T, Verheij M, Allum W, Cunningham D, Cervantes A, Arnold D: Gastric cancer: ESMO-ESSO-ESTRO clinical practice guidelines for diagnosis, treatment and follow-up. European journal of surgical oncology 2014; 40:584-91.

10. Liu D, Lu M, Li J, Yang Z, Feng Q, Zhou M et al: The patterns and timing of recurrence 2016; 14:305

11. Paoletti X, Oba K, Burzykowski T, Michiels S, Ohashi Y, Pignon JP et al: Benefit of Adjuvant Chemotherapy for Resectable Gastric Cancer A Meta-analysis. JAMA-J Am Med Assoc chemotherapy for gastric cancer with S-1, an oral fluoropyrimidine. New England Journal 
of Medicine 2007; 357:1810-20.

13. Sasako M, Sakuramoto S, Katai H, Kinoshita T, Furukawa H, Yamaguchi T et al: Five-Year Outcomes of a Randomized Phase III Trial Comparing Adjuvant Chemotherapy With S-1 Versus Surgery Alone in Stage II or III Gastric Cancer. Journal of Clinical Oncology 2011; 29:4387-93.

14. Takahari D, Hamaguchi T, Yoshimura K, Katai H, Ito S, Fuse $\mathrm{N}$ et al: Feasibility study of Pharmacol 2011; 67:1423-8.

15. Takahari D, Hamaguchi T, Yoshimura K, Katai H, Ito S, Fuse $\mathrm{N}$ et al: Survival analysis of

16. Shitara K, Chin K, Yoshikawa T, Katai H, Terashima M, Ito S et al: Phase II study of adjuvant

17. Yoshida K, Kodera Y, Kochi M, Ichikawa W, Kakeji Y, Sano T et al: Addition of Docetaxel to

18. Tamura S, Fujitani K, Kimura Y, Tsuji T, Matsuyama J, lijima S et al: Phase II feasibility study of adjuvant S-1 plus docetaxel for stage III gastric cancer patients after curative D2 gastrectomy. Oncology 2011; 80:296-300.

Fujitani K, Tamura S, Kimura Y, Tsuji T, Matsuyama J, lijima S et al: Three-year outcomes 
of a phase II study of adjuvant chemotherapy with S-1 plus docetaxel for stage III gastric cancer after curative D2 gastrectomy. Gastric Cancer 2014; 17:348-53.

20. Nakamura Y, Yamanaka T, Chin K, Cho H, Katai H, Terashima M et al: Survival Outcomes of Two Phase 2 Studies of Adjuvant Chemotherapy with S-1 Plus Oxaliplatin or Capecitabine Plus Oxaliplatin for Patients with Gastric Cancer After D2 Gastrectomy. Ann

21. Wang G, Zhao J, Song Y, Zhang W, Sun Y, Zhou A et al: Phase II study of adjuvant Cancer 2018; 18:547. chemotherapy with S1 plus oxaliplatin for Chinese patients with gastric cancer. BMC

23. Noh SH, Park SR, Yang H-K, Chung HC, Chung I-J, Kim S-W et al: Adjuvant capecitabine combined with S-1 (SOX) versus postoperative chemotherapy of SOX or oxaliplatin with capecitabine (XELOX) in locally advanced gastric adenocarcinoma with D2 gastrectomy: 
26. Grothey A, Sobrero AF, Shields AF, Yoshino T, Paul J, Taieb J et al: Duration of Adjuvant Chemotherapy for Stage III Colon Cancer. New England Journal of Medicine 2018; 378:1177-88.

27. Yoshikawa T, Terashima M, Mizusawa J, Nunobe S, Nishida Y, Yamada T et al: Four courses versus eight courses of adjuvant S-1 for patients with stage II gastric cancer (JCOG1104 OPAS-1 ): an open-label, phase 3, non-inferiority, randomised trial. Lancet Gastroenterology \& Hepatology 2019; 4:208-16.

28. Wang SM, Zheng RS, Zhang SW, Zeng HM, Chen R, Sun KX et al: Epidemiological characteristics of gastric cancer in China, 2015. Zhonghua liu xing bing xue za zhi 2019; 40:1517-21.

29. Jatoi A, Foster NR, Egner JR, Burch PA, Stella PJ, Rubin J et al: Older versus younger patients with metastatic adenocarcinoma of the esophagus, gastroesophageal junction, and stomach: a pooled analysis of eight consecutive North Central Cancer Treatment Group (NCCTG) trials. International Journal of Oncology 2010; 36:601-6.

30. Kodera Y, Ishiyama A, Yoshikawa T, Kinoshita T, Ito S, Yokoyama H et al: A feasibility study of postoperative chemotherapy with S-1 and cisplatin (CDDP) for gastric carcinoma (CCOG0703). Gastric Cancer 2010; 13:197-203.

31. Kurimoto K, Ishigure K, Mochizuki Y, Ishiyama A, Matsui T, Ito S et al: A feasibility study of postoperative chemotherapy with S-1 and cisplatin (CDDP) for stage III/IV gastric cancer (CCOG 1106). Gastric Cancer 2015; 18:354-9.

32. Yoshida K: Confirmed three-year RFS and OS of the randomized trial of adjuvant S-1 versus S-1 plus docetaxel after curative resection of pStage III gastric cancer 
33. Kim IH, Park SS, Lee CM, Kim MC, Kwon IK, Min JS et al: Efficacy of Adjuvant S-1 Versus XELOX Chemotherapy for Patients with Gastric Cancer After D2 Lymph Node Dissection: A Retrospective, Multi-Center Observational Study. Ann Surg Oncol 2018; 25:1176-83.

34. Cho JH, Lim JY, Cho JY: Comparison of capecitabine and oxaliplatin with S-1 as adjuvant chemotherapy in stage III gastric cancer after D2 gastrectomy. PLoS One 2017;

35. Choi KS, Jun JK, Suh M, Park B, Noh DK, Song SH et al: Effect of endoscopy screening on stage at gastric cancer diagnosis: results of the National Cancer Screening Programme in Korea. Br J Cancer 2015; 112:608-12.

36. Yamamoto K, Tsujinaka T, Takahashi T, Sato S, Nishiguchi Y, Nakashima Y et al: Impact of Oncol 2015; 22:232-9.

37. Hamashima C, Ogoshi K, Okamoto M, Shabana M, Kishimoto T, Fukao A: A CommunityBased, Case-Control Study Evaluating Mortality Reduction from Gastric Cancer by gastrectomy for adenocarcinoma of the gastric antrum. A French prospective controlled study. Annals of Surgery 1989; 209:162-6. 
gastrectomy for gastric cancer - Five-year survival rates in a multicenter randomized Italian trial. Annals of Surgery 1999; 230:170-8.

413 40. Bozzetti F, Marubini E, Bonfanti G, Miceli R, Piano C, Crose N et al: Total versus subtotal gastrectomy - Surgical morbidity and mortality rates in a multicenter Italian randomized trial. Annals of Surgery 1997; 226:613-20.

41. Clark CJ, Thirlby RC, Picozzi V, Jr., Schembre DB, Cummings FP, Lin E: Current problems in surgery: gastric cancer. Current Problems in Surgery 2006; 43:566-670.

42. Robertson CS, Chung SCS, Woods SDS, Griffin SM, Raimes SA, Lau JTF et al: A prospective

45. Cunningham D, Allum WH, Stenning SP, Thompson JN, Van de Velde CJ, Nicolson M et al: Perioperative chemotherapy versus surgery alone for resectable gastroesophageal cancer. New England Journal of Medicine 2006; 355:11-20.

46. Ychou M, Boige V, Pignon JP, Conroy $\mathrm{T}$, Bouche $\mathrm{O}$, Lebreton $\mathrm{G}$ et al: Perioperative chemotherapy compared with surgery alone for resectable gastroesophageal adenocarcinoma: an FNCLCC and FFCD multicenter phase III trial. Journal of Clinical 
434 47. Wang X, Li S, Xie T, Lu Y, Guo X, Lin C: Early results of the randomized, multicenter, controlled evaluation of S-1 and oxaliplatin as neoadjuvant chemotherapy for Chinese advanced gastric cancer patients (RESONANCE Trial). Journal of Clinical Oncology 2020; 38 suppl 4:280.

48. Dhar DK, Kubota H, Tachibana M, Kinugasa S, Masunaga R, Shibakita M et al: Prognosis Neoadjuvant chemotherapy with S-1 and cisplatin followed by D2 gastrectomy with para- 
aortic lymph node dissection for gastric cancer with extensive lymph node metastasis. British Journal of Surgery 2014; 101:653-60.

54. Ito S, Sano T, Mizusawa J, Takahari D, Katayama H, Katai $\mathrm{H}$ et al: A phase II study of preoperative chemotherapy with docetaxel, cisplatin, and S-1 followed by gastrectomy with D2 plus para-aortic lymph node dissection for gastric cancer with extensive lymph node metastasis: JCOG1002. Gastric Cancer 2017; 20:322-31.

55. Morita S, Ito S, Sano T, Takahari D, Katayama H, Mizusawa J et al: Long-term outcome of preoperative docetaxel with cisplatin plus S-1 therapy for advanced gastric cancer with extensive nodal metastasis (JCOG1002). Journal of Clinical Oncology 2019; 37 suppl 4:141.

56. Al-Batran S-E, Homann N, Pauligk C, Goetze TO, Meiler J, Kasper S et al: Perioperative chemotherapy with fluorouracil plus leucovorin, oxaliplatin, and docetaxel versus fluorouracil or capecitabine plus cisplatin and epirubicin for locally advanced, resectable gastric or gastro-oesophageal junction adenocarcinoma (FLOT4): a randomised, phase 2/3 trial. The Lancet 2019; 393:1948-57.

57. Al-Batran S-E, Hofheinz RD, Pauligk C, Kopp H-G, Haag GM, Luley KB et al: Histopathological regression after neoadjuvant docetaxel, oxaliplatin, fluorouracil, and leucovorin versus epirubicin, cisplatin, and fluorouracil or capecitabine in patients with resectable gastric or gastro-oesophageal junction adenocarcinoma (FLOT4-AIO): results from the phase 2 part of a multicentre, open-label, randomised phase 2/3 trial. The Lancet Oncology 2016; 17:1697-708.

58. Al-Batran SE, Homann N, Schmalenberg H, Knopp HG, Georg: Perioperative chemotherapy with docetaxel, oxaliplatin, and fluorouracil/leucovorin (FLOT) versus 
epirubicin, cisplatin, and fluorouracil or capecitabine (ECF/ECX) for resectable gastric or gastroesophageal junction (GEJ) adenocarcinoma (FLOT4-AIO): A multicenter, randomized phase 3 trial. Journal of Clinical Oncology 2017; 35 suppl 15:4004.

480 59. Wang K, Ren Y, Ma Z, Li F, Cheng X, Xiao J et al: Docetaxel, oxaliplatin, leucovorin, and 5fluorouracil (FLOT) as preoperative and postoperative chemotherapy compared with surgery followed by chemotherapy for patients with locally advanced gastric cancer: a propensity score-based analysis. Cancer Management and Research 2019; 11:3009-20. 
Figures

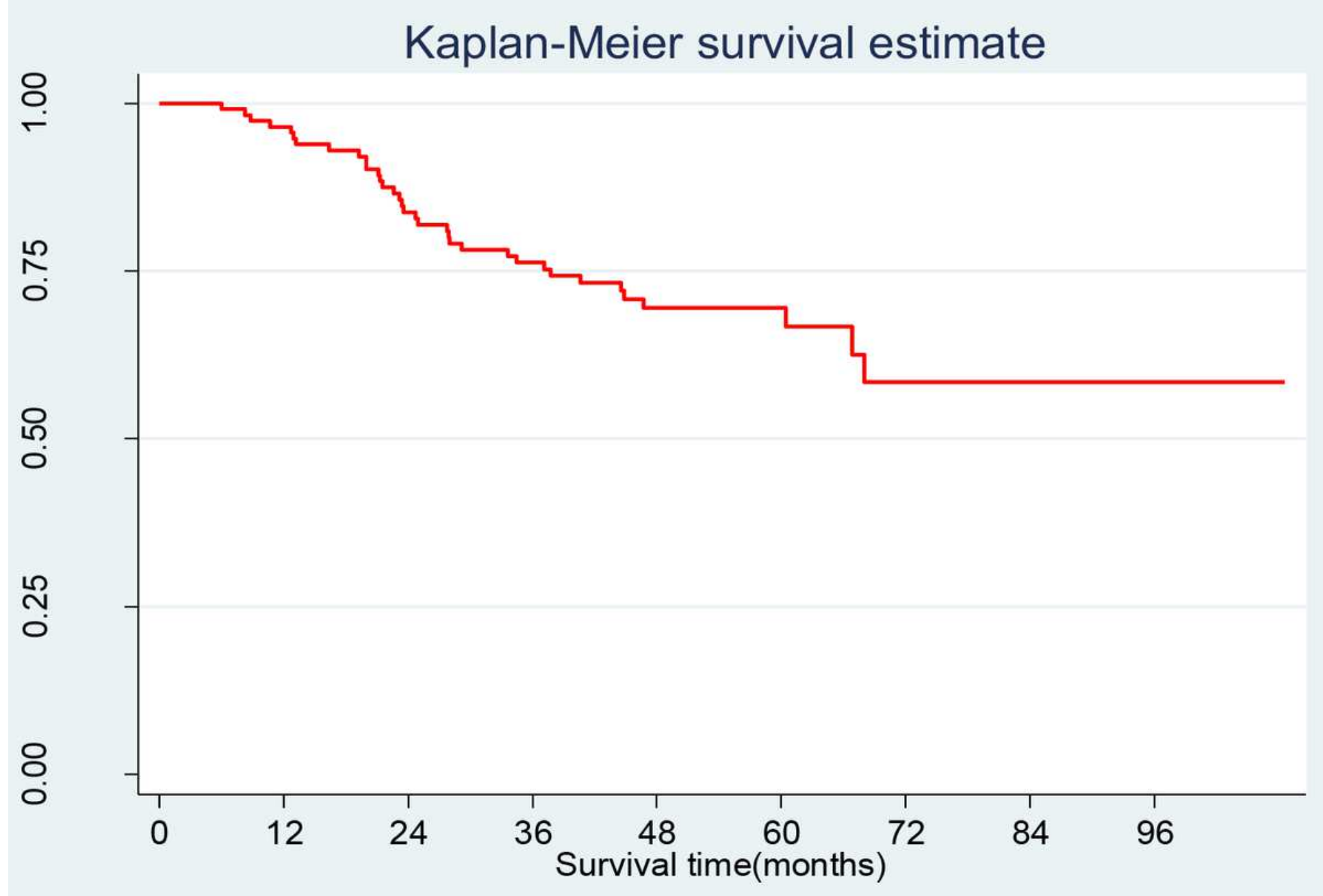

Figure 1

The Kaplan-Meier curves for overall survival curve in all patients 


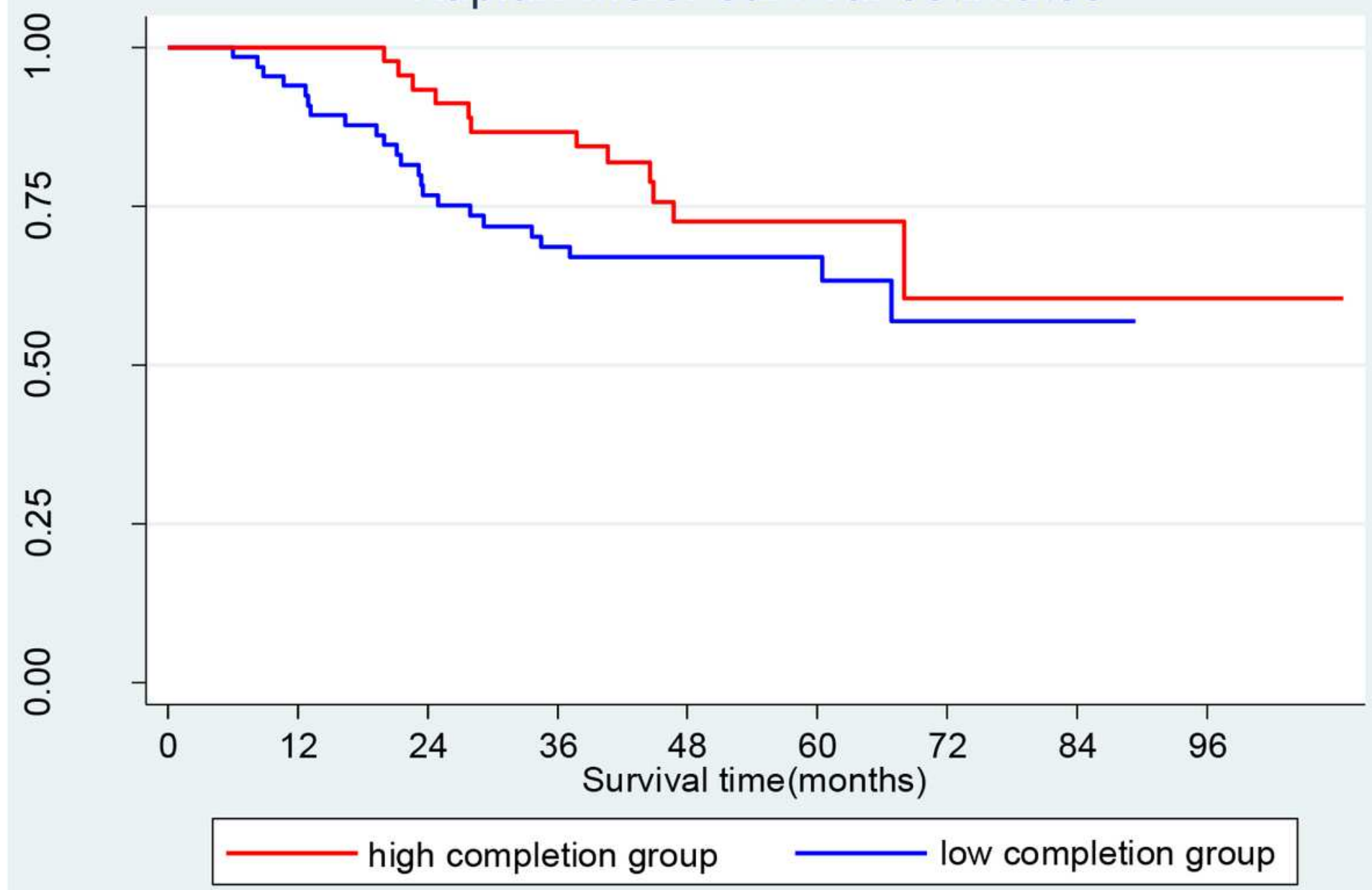

Figure 2

OS in the high completion group and low completion group 


\section{Kaplan-Meier survival estimates}

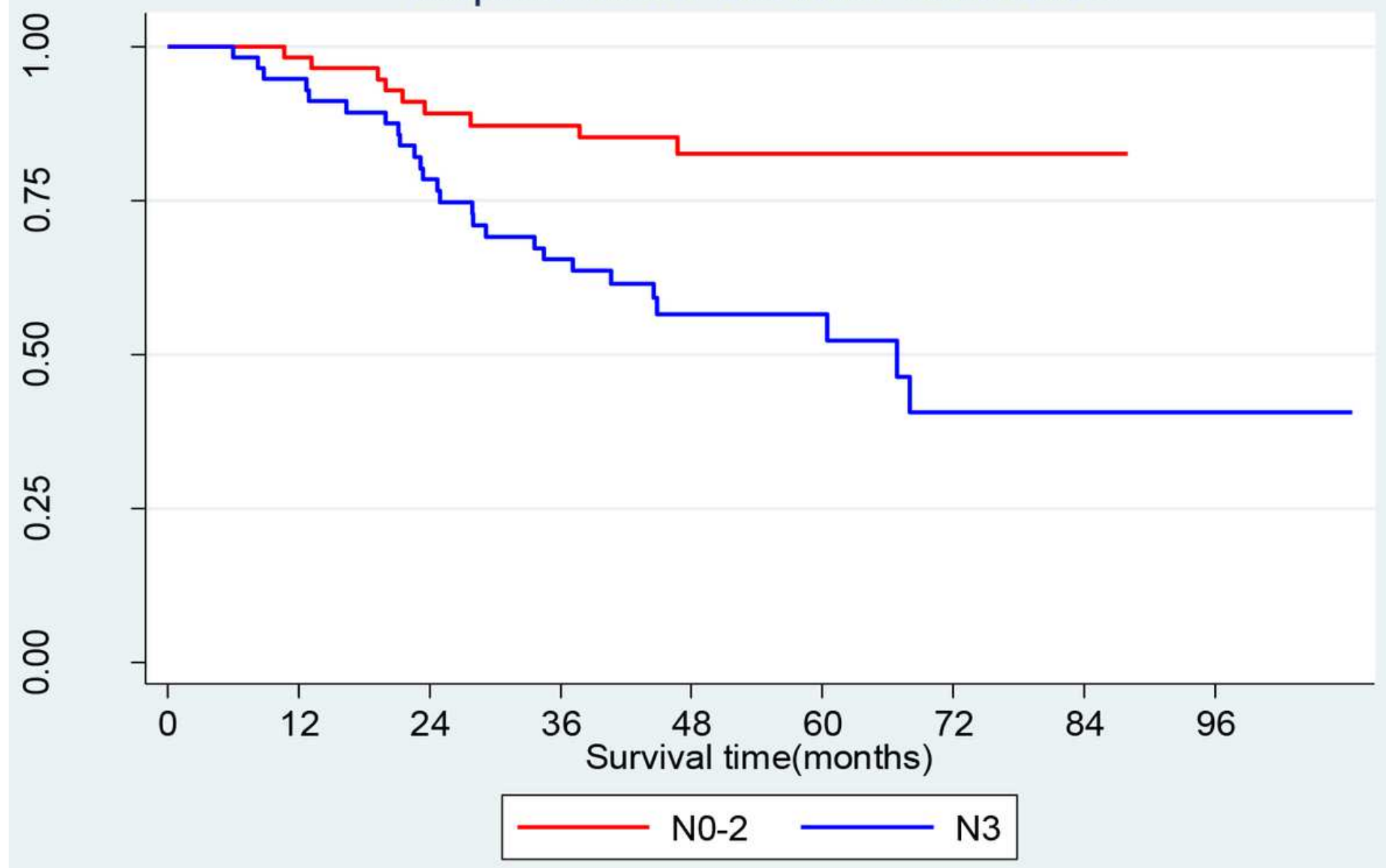

Figure 3

OS in patients with pNO-2 and pN3 


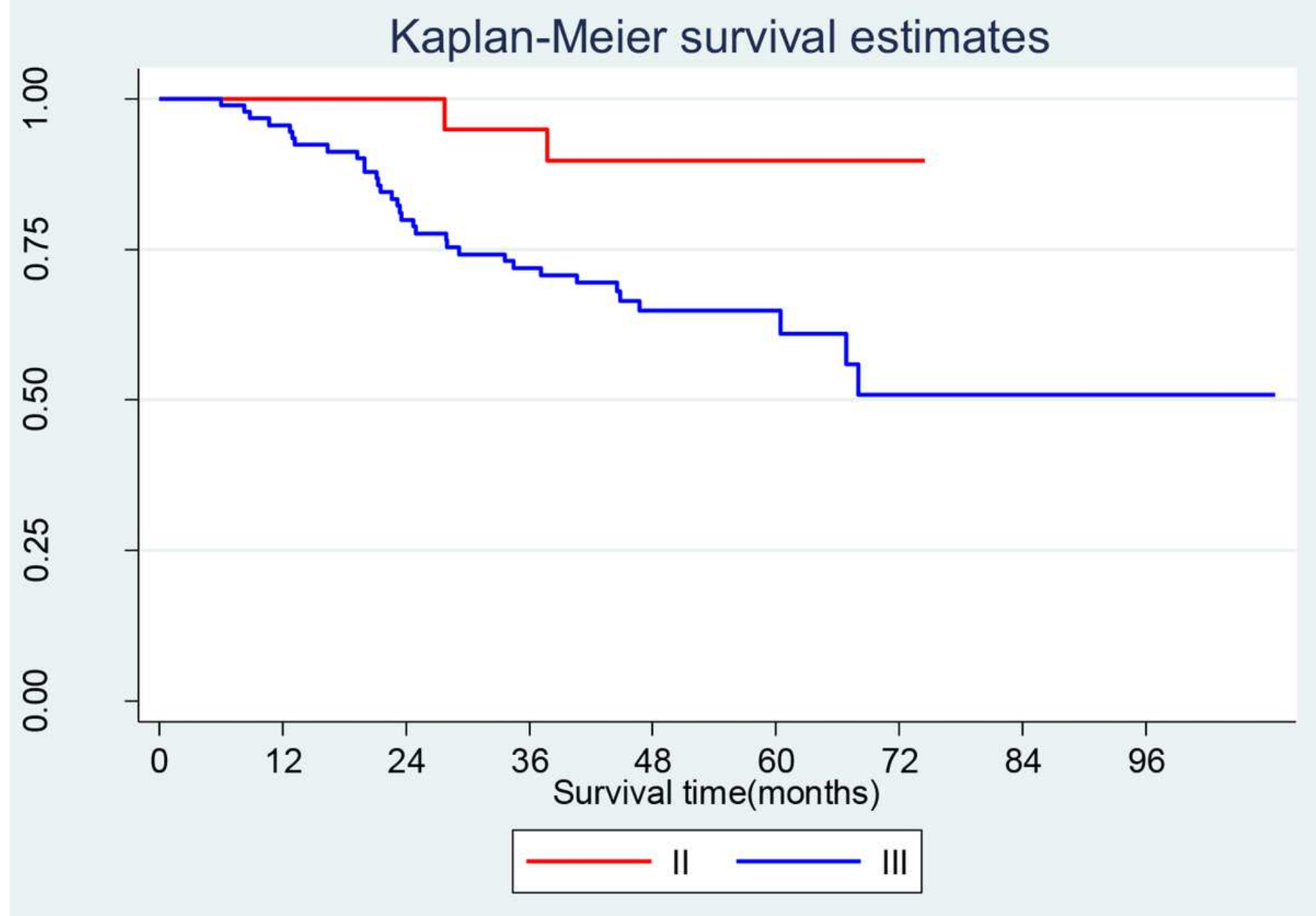

Figure 4

OS in patients with pTNM II stage and pTNM III stage 


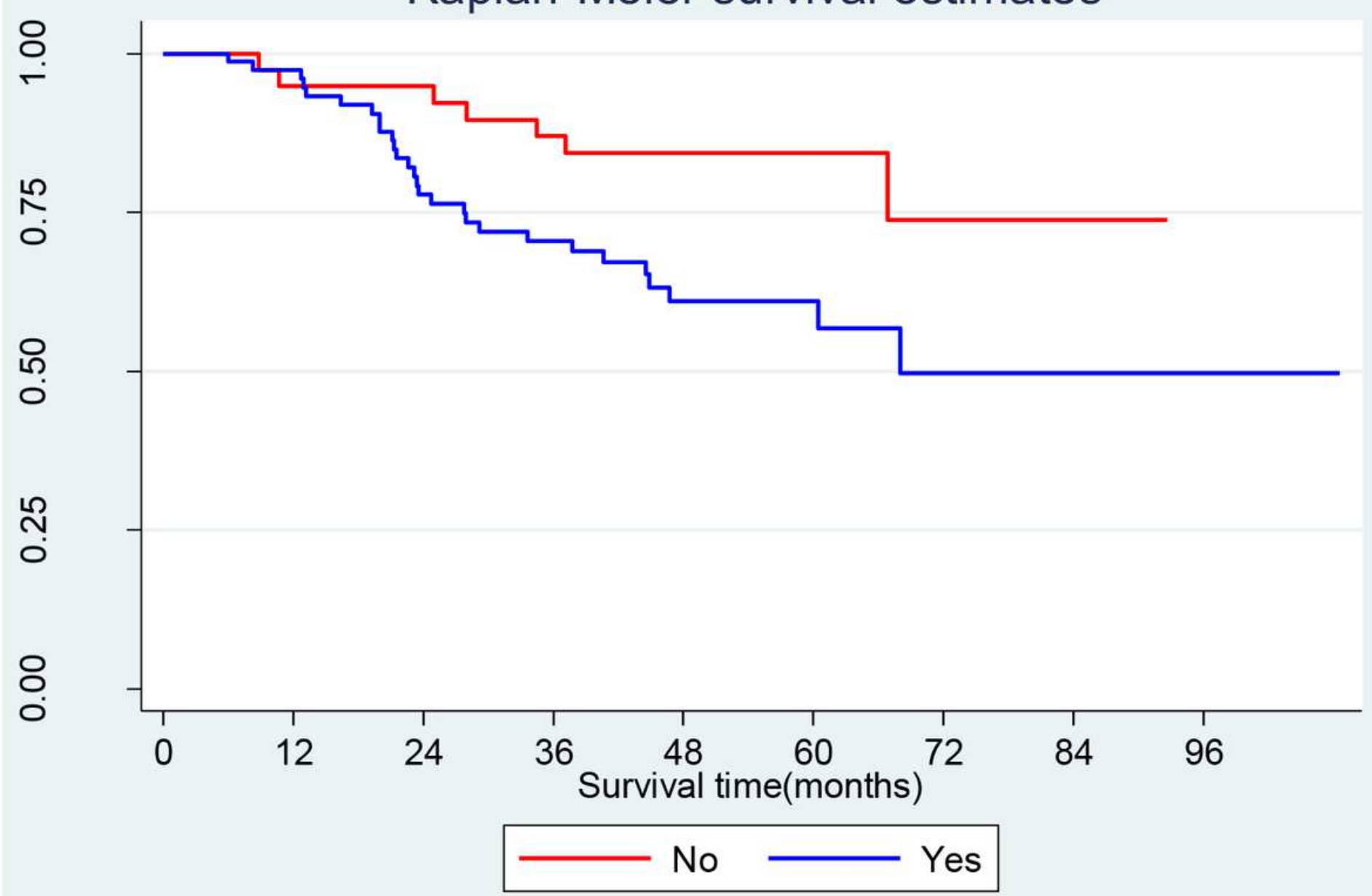

Figure 5

OS in patients with nerve invasion and non-nerve invasion

\section{Supplementary Files}

This is a list of supplementary files associated with this preprint. Click to download.

- table1.pdf

- table2.pdf

- table3.pdf

- table4.pdf

- table5.pdf

- table6.pdf

- table7.pdf 